Motor unit number estimation (MUNE) is an electrophysical technique to estimate the number of motor units innervating a muscle or muscle group. MUNE may be useful as a measure of progression of lower motor neuron loss in amyotrophic lateral sclerosis (ALS). Several methods of MUNE have been developed. The spike-triggered averaging method can be readily performed on EMG machines with signal averaging capabilities and is suitable for estimating the number of motor neurons innervating proximal muscles. We have used MUNE as a measure of disease state in a drug efficacy trial for ALS. From our experience with this method we have identified sources of error which can affect MUNE accuracy. We have investigated these sources and report their effect on MUNE. (c) 1995 John Wiley \& Sons, Inc.

Key words: motor unit number estimation - EMG - ALS

\title{
SOURCES OF ERROR IN THE SPIKE-TRIGGERED AVERAGING METHOD OF MOTOR UNIT NUMBER ESTIMATION (MUNE)
}

MARK B. BROMBERG, MD, PhD, and JUDITH L. ABRAMS, PhD

Amyotrophic lateral sclerosis (ALS) is a disease for which it would be useful to know the number of motor units innervating a muscle or muscle group as an aid to establishing the diagnosis, for following the natural course of the disease, or for assessing the efficacy of a drug in a therapeutic trial. It is not possible at this time to obtain the true number of motor units innervating a muscle, ${ }^{17}$ but there are noninvasive electrophysiological techniques available which can estimate this number, and these techniques are termed motor unit number estimation (MUNE).

We have investigated the use of MUNE as an outcome measure in a drug efficacy trial for ALS. ${ }^{6}$ A number of MUNE methods have been described, ${ }^{10,12,17,19}$ and we have chosen the spiketriggered method. ${ }^{9}$ This method does not rely on the availability of special EMG machine software, and can be carried out on any machine which has

From the Departments of Neurology (Dr. Bromberg) and Biostatistics (Dr. Abrams), The University of Michigan, Ann Arbor, Michigan.

Acknowledgments: We wish to thank Dr. William Brown and Dr. James Albers for their encouragement and review of the manuscript. Supported in part by grants from the Muscular Dystrophy Association, the FDA (FD$\mathrm{R}-000512-02$ ), and the NIH (MO 1-RR00042)

Address reprint requests to Dr. M.B. Bromberg. Department of Neurology The University of Utah, 50 North Medical Drive, Salt Lake City, UT 84132

Accepted for publication May 1, 1995.

CCC 0148-639X/95/101139-08

(c) 1995 John Wiley \& Sons, Inc signal averaging capabilities. The spike-triggered averaging method can be readily applied to proximal muscles which are routinely included in quantitative isometric strength testing, such as the Tufts Quantitative Neurologic Examination (TQNE), ${ }^{1,2}$ and permits a comparison between MUNE values and strength measurements. ${ }^{5}$

From our experience with MUNE, ${ }^{4-6}$ a number of sources of error have been encountered that are related to the spike-triggered method, which if unrecognized could affect the accuracy of MUNE. In this report we describe our investigations of these sources of error for this method. There are general assumptions and methodological limitations common to all MUNE methods which have been addressed elsewhere ${ }^{8,9,12,17,20}$ and will not be formally considered in this report.

\section{METHODS}

Elbow flexor muscles were studied in 10 normal and 15 ALS subjects. All electrodiagnostic studies were performed on Nicolet Viking EMG equipment (Nicolet Instrument Corp., Madison, WI) using routine nerve conduction and averaging software commercially available from the manufacturer. MUNE was carried out in the bicepsbrachialis muscle group by a modification of spiketriggering averaging. ${ }^{9}$ The stigmatic electrode was $7 \times 80 \mathrm{~mm}$ silver strip placed transversely across the motor point of the biceps brachii muscle and 


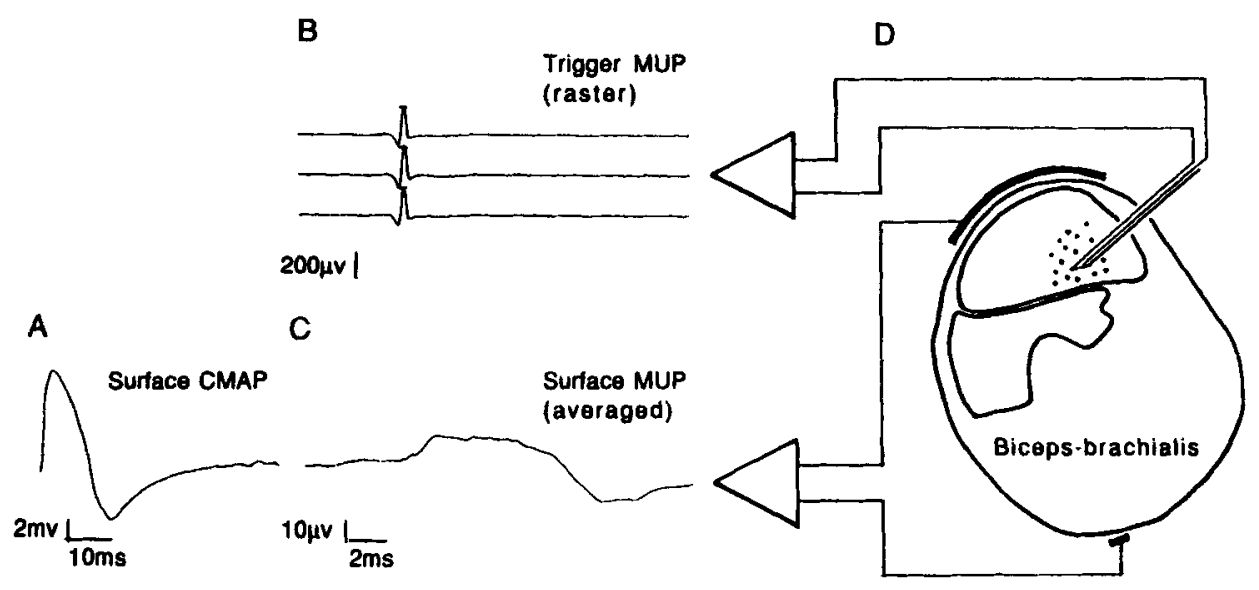

FIGURE 1. Recording arrangement for MUNE in biceps-brachialis muscles. (A) CMAP following supramaximal musculocutaneous nerve stimulation. (B) Triggering motor unit action potentials shown in raster mode. (C) Averaged S-MUP. (D) Cross section of muscles with intramuscular trigger electrode, surface strip electrode, and reference electrode.

the reference electrode was a disk placed over the olecranon process (Fig. 1). The maximal compound muscle action potential (CMAP) from the biceps-brachialis muscle group was obtained by percutaneous electrical stimulation of the musculocutaneous nerve in the axilla. A single-fiber macro-EMG or concentric EMG recording electrode was introduced into the biceps brachii muscle $3-5 \mathrm{~cm}$ distal to the stigmatic electrode and used to isolate the triggering motor unit spike potentials, which were generated by weak voluntary muscle contractions. The surface motor unit action potential (S-MUP) from the triggering motor unit was recorded by the strip electrode. The stability of

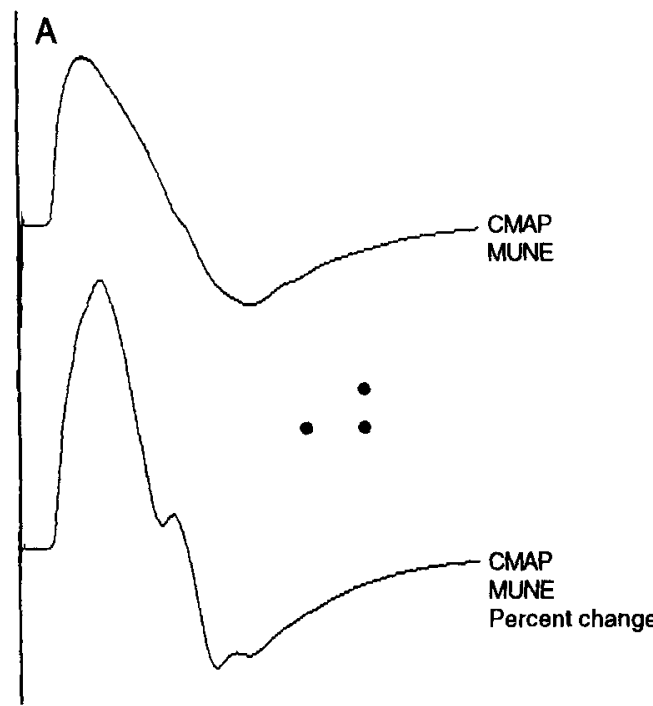

the triggering potential during the recording epoch was monitored by a raster display. Each S-MUP obtained for measurement was averaged approximately 200 times. Fifteen S-MUPs were obtained from each muscle. The triggering motor unit spike potentials were recorded at five different depths and from three penetrations at medial, middle, and lateral sites across the muscle. The MUNE was calculated as the peak-to-peak CMAP amplitude divided by the mean peak-to-peak amplitude of 15 averaged S-MUPs. A S-MUP was discarded only if it was identical in shape and amplitude to the preceding potential.

The following methods were used to study par-

\section{B}

\begin{tabular}{ccc}
$\begin{array}{c}\text { Negative peak } \\
\text { amplitude (mv) }\end{array}$ & $\begin{array}{c}\text { Peak-to-peak } \\
\text { amplitude (mv) }\end{array}$ & $\begin{array}{c}\text { Negative peak } \\
\text { area (mv sec) }\end{array}$ \\
\hline 15.4 & 22.9 & 112.4 \\
766 & 552 & 876
\end{tabular}

$\begin{array}{ccc}24.8 & 35.9 & 157.4 \\ 1234 & 865 & 1235 \\ 161 & 157 & 141\end{array}$

FIGURE 2. Effects of volume conduction on biceps-brachialis CMAP amplitude and area. (A) Upper trace, normal CMAP; bottom trace, inflated CMAP due to volume conduction from distant muscles. (B) Table of CMAP amplitude and area measurements and resultant MUNE values. Time: $5 \mathrm{~ms} / \mathrm{div}$, voltage $5 \mathrm{mV} / \mathrm{div}$. 


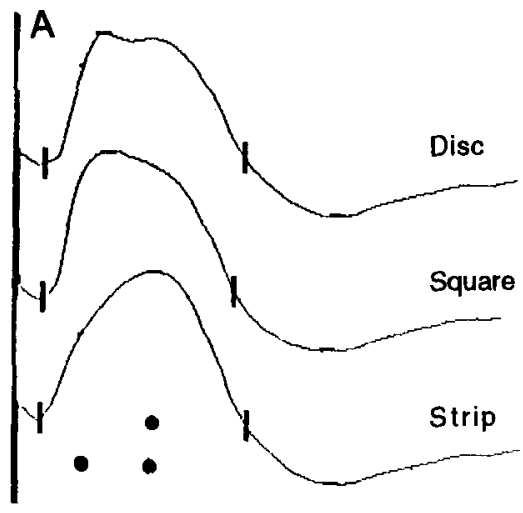

B

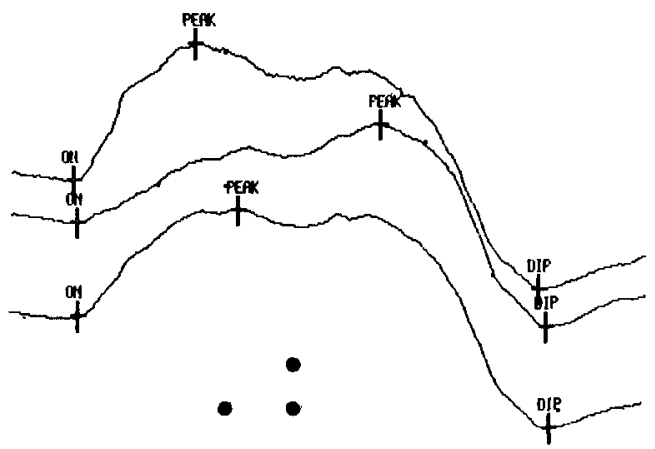

FIGURE 3. Effects of stigmatic electrode size, shape, and placement on the CMAP and S-MUP. (A) CMAP wave forms; top recorded from $7 \mathrm{~mm}$ diameter disk electrode, middle recorded from $20 \mathrm{~mm}$ square electrode, lower recorded from $7 \times 80 \mathrm{~mm}$ strip electrode. Time: $5 \mathrm{~ms} / \mathrm{div}$, voltage $2 \mathrm{mV} / \mathrm{div}$. (B) Representative S-MUP wave forms recorded from same electrodes. The MUNE values derived from the three recording electrodes were identical. Time: $2 \mathrm{~ms} / \mathrm{div}$, voltage $20 \mu \mathrm{V} / \mathrm{div}$.
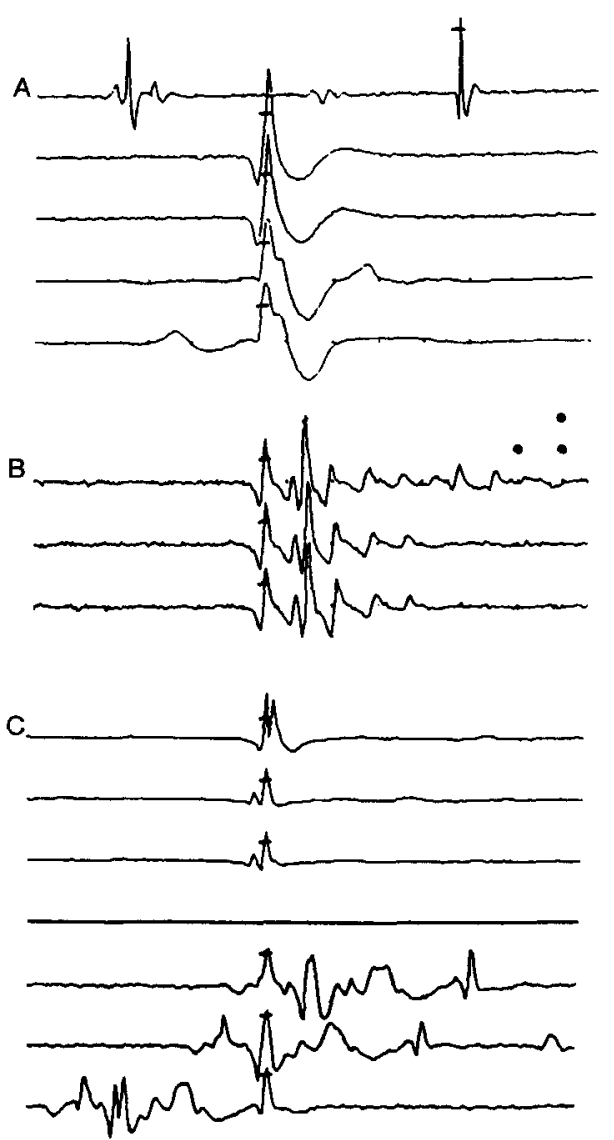

FIGURE 4. Abnormal trigger potentials. (A) Spurious trigger potentials; top trace, continuous time; lower trace, raster format. Distant motor unit potentials occasionally superimpose with trigger potentials. (B) Trigger potentials in raster format showing occasional blocking of components. (C) Time shifts of the trigger point within the trigger potential due to amplitude changes of spike components within the trigger potential. Top raster: example of small temporal shift. Bottom raster: example of large temporal shift. Time: $2 \mathrm{~ms} / \mathrm{div}$, voltage $20 \mu v /$ div. ticular sources of error and their effects on MUNE accuracy. (1) Contamination of the biceps brachii CMAP by volume conduction from other muscles was induced by moving the stimulating electrode to activate other nerves coursing through the axilla (Fig. 2). (2) The effects of stigmatic electrode size, shape, and placement was studied in several subjects by applying additional stigmatic electrodes of circular or square shape near to the strip electrode (Fig. 3). (3) The effects of spurious trigger potentials (Fig. 4) on the averaged S-MUP amplitude were investigated by calculating the percentage change in the averaged amplitude produced by adding to the average various numbers of spurious potentials of different sizes (Table 1). (4) To determine the physiologic range of amplitudes of spurious trigger potentials, the distribution of S-MUP amplitudes was assessed in 10 normal subjects and 15 subjects with ALS (Fig. 5). (5) The effect on the averaged S-MUP amplitude of tem-
Table 1. Percentage change in the averaged amplitude of a S-MUP when various numbers of spurious potentials of different relative amplitudes are added to a simple running average of 200 potentials.

\begin{tabular}{cccc}
\hline & \multicolumn{3}{c}{$\begin{array}{c}\text { Number of spurious potentials } \\
\text { added to the average }\end{array}$} \\
\cline { 2 - 4 } Relative amplitude & $0.5 \%$ & $2.5 \%$ & $5 \%$ \\
of spurious signals & $(1 / 200)$ & $(5 / 200)$ & $(10 / 200)$ \\
\hline 0.5 & 99.8 & 99 & 98 \\
1 & 100 & 100 & 100 \\
2 & 100.5 & 102 & 105 \\
5 & 102 & 110 & 119 \\
10 & 104 & 122 & 143 \\
20 & 109 & 143 & \\
30 & 114 & & \\
\hline
\end{tabular}




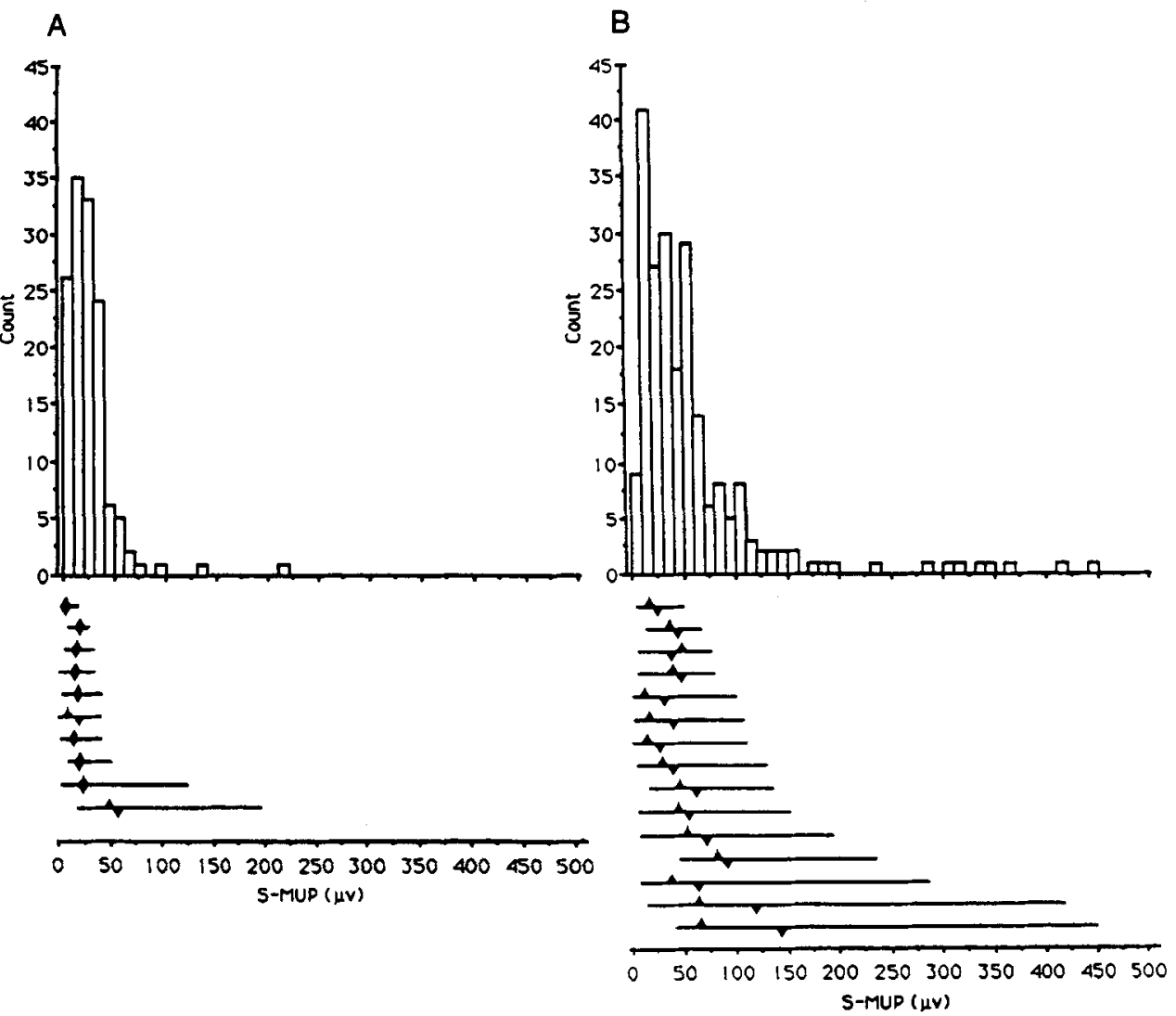

FIGURE 5. Distribution of S-MUP amplitudes. (A) Normal subjects. (B) ALS subjects. Top: cumulative amplitude histogram. Bottom: range of amplitudes for individual subjects showing mean (triangles above the line) and median values (triangles below line).

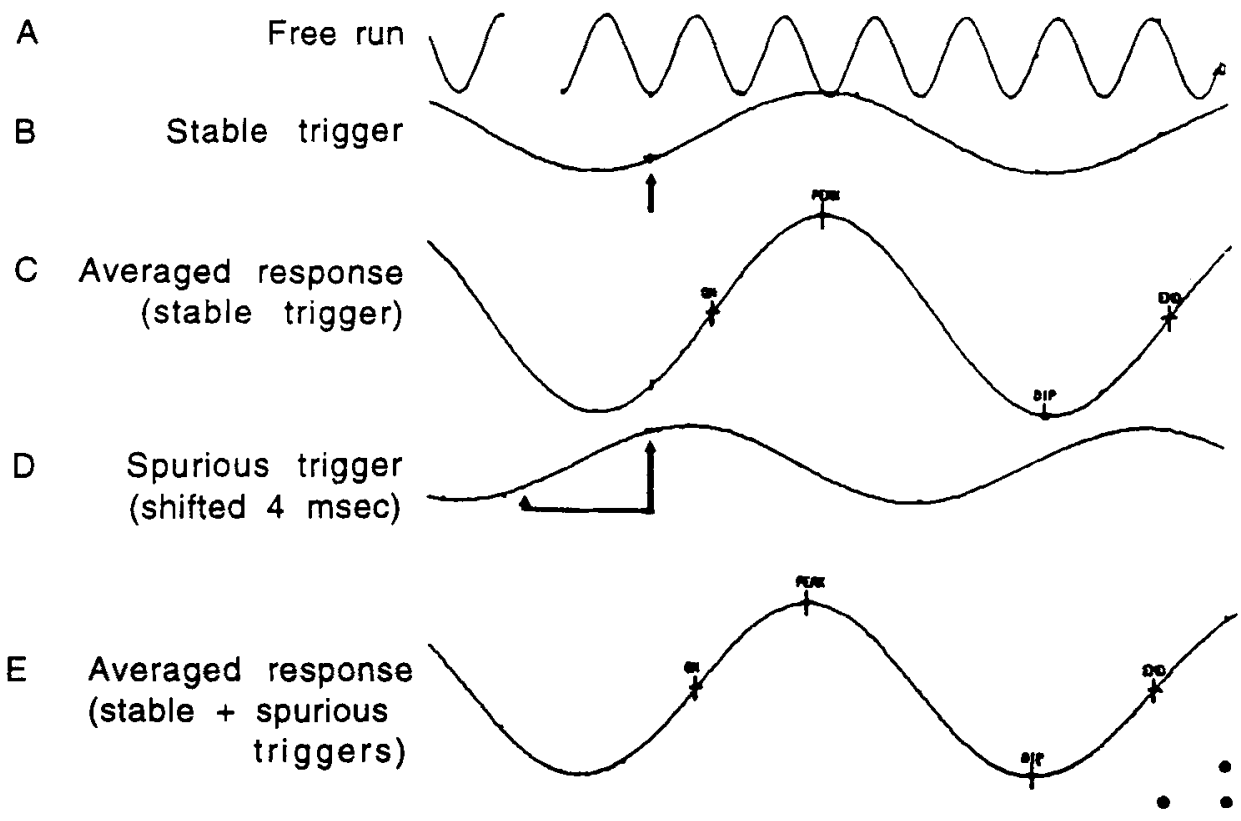

FIGURE 6. Simulation of the effects of time shifts of the trigger point within the trigger potential on the amplitude of the averaged S-MUP. (A) Sine wave signal. Interruption is an artifact. (B) Stable trigger leading to accurate averaged response (C). (D) Spurious trigger shifted $4 \mathrm{~ms}$ leading to reduced average response (E). Time: trace $A 10 \mathrm{~ms} / \mathrm{div}$, traces $B-E 2 \mathrm{~ms} /$ div. Voltage: traces $A, B, D$ $500 \mathrm{mV} /$ div, traces C,E $500 \mu \mathrm{V} /$ div. 
poral shifting of the trigger point within the triggering motor unit due to instability was investigated in a simulation model. The shape of a S-MUP was modeled by a sine wave with a frequency of approximately $63 \mathrm{~Hz}$ derived from a waveform generator (Fig. 6). The modeled sine waves were averaged in an identical manner as that described for S-MUPs obtained from subjects. The effects of temporal shifting of the trigger were studied by adding to the accumulating average different numbers of sine waves which were shifted either $2 \mathrm{~ms}$ or $4 \mathrm{~ms}$ earlier (Table 2). (6) The effect of the depth of motor units within the muscle on S-MUP amplitude was assessed in 21 penetrations in ALS subjects and in five penetrations in normal subjects. In these penetrations the triggering units were isolated sequentially as the electrode was advanced through the full depth of the muscle. In the ALS subjects, a single-fiber macro-EMG electrode was used as the intramuscular triggering electrode. This permitted simultaneous recording of a motor unit from intramuscular and surface viewing points and a comparison of their amplitudes.

\section{RESULtS}

MUNE values are calculated from the ratio of the maximal CMAP response to the average single motor unit response. Errors may affect either the numerator or denominator of this ratio.

Errors in Recording the Compound Muscle Action Potential. Volume Conduction. In attempting to record the maximal CMAP of the muscle under study, potentials from remote muscles, whose motor units will not be recorded by the intramuscular electrode used to collect S-MUPs, may contaminate the CMAP. Volume conduction of electrical activity from these distant muscles may occur as a consequence of inadvertent stimulation of neighboring nerves.

Table 2. Percentage changes in the averaged amplitude of a S-MUP when various numbers of trigger potentials with shifting trigger points are added to a simple running average of 200 potentials.

\begin{tabular}{lccc}
\hline & \multicolumn{3}{c}{$\begin{array}{c}\text { Number of spurious potentials } \\
\text { added to the average }\end{array}$} \\
\cline { 2 - 4 } Trigger & $5 \%$ & $10 \%$ & $20 \%$ \\
time shift & $(10 / 200)$ & $(20 / 200)$ & $(40 / 200)$ \\
\hline $2 \mathrm{~ms}$ & 99 & 97 & 95 \\
$4 \mathrm{~ms}$ & 95 & 89 & 82 \\
\hline
\end{tabular}

The musculocutaneous nerve is in close proximity to the ulnar and median nerves in the axilla and arm, and contributions from the respective forearm flexor muscles may add to the bicepsbrachialis CMAP. Volume conduction can be detected by the emergence of distortions in the expected CMAP wave form (Fig. 2), by observing contraction of additional muscles, or by reports by the subjects of paresthesias in the appropriate nerve distributions. ${ }^{9}$ The magnitude of the resultant MUNE error is dependent upon where the distortions occur in the CMAP wave form, and whether amplitude or area are the units of measure used in the MUNE ratio. Examples in Figure 2 of erroneously large CMAPs yield MUNE values up to $161 \%$ of normal.

The location of the surface recording electrode over the muscle will influence the amplitude and configuration of the CMAP, but will also influence the S-MUP in a similar manner. ${ }^{13}$ Simultaneous MUNE determinations were made with electrodes of different shapes and positions over the biceps muscle. For most determinations, the ratios from different electrodes were identical (Fig. 3).

Errors In Recording the Surface Motor Unit Potential. Spurious Trigger Potentials. Spurious trigger potentials may occur when the trigger threshold is crossed by either a different voluntary motor unit potential or by an involuntary fasciculation potential. The motor unit potential associated with the spurious potential will be included in the accumulating average (Fig. 4). The final amplitude of the contaminated averaged S-MUP potential will depend upon the surface amplitude of the spurious motor unit potentials, the number of spurious motor unit potentials included in the average, and the total number of potentials that are averaged. The surface amplitude of a spurious potential cannot be determined from inspection of the S-MUP because its effect will be obscured within the accumulating average.

The effects of spurious potentials on the averaged S-MUP, however, can be calculated (Table 1). Calculations show that low amplitude spurious motor unit potentials are less influential than high amplitude potentials. A single spurious potential whose amplitude is up to ten times the amplitude of the triggering potential will inflate the averaged amplitude up to $104 \%$, an acceptable level of error. Spurious motor units whose amplitudes are less than ten times the averaged S-MUP potential become problematic when more than one is included in the average, and inclusion of more than ten spu- 
rious potentials of twice the average amplitude, or more than two potentials of five times the amplitude will inflate the average to over $105 \%$, a problematic level of error.

To determine the physiologic range of the amplitudes of spurious trigger potentials, the distribution of S-MUP amplitudes was assessed in 10 normal subjects and 15 subjects with ALS. For individual normal subjects, S-MUP amplitudes were distributed across a narrow but skewed range which spanned one and rarely two orders of magnitude (Fig. 5). Individual ALS subjects may have S-MUP of large amplitude due to collateral reinnervation, and the range of amplitudes was shifted to larger values in subjects with more advanced denervation and reinnervation. Despite the shift to larger values, the S-MUP amplitude range remained one to two orders of magnitude in breadth in ALS subjects.

Trigger Potential Component Blocking. Tenuous neuromuscular junction transmission, manifest by abnormal jitter and blocking, is common in ALS subjects $^{18}$ and results in discharge-to-discharge variability in the number of fibers contributing to the S-MUP being averaged (Fig. 4). Transmission variability applies to all newly reinnervated muscle fibers in the motor unit, and the amplitude of the S-MUP will vary from discharge to discharge. An estimate of transmission failure in the triggering potential can be monitored by the raster display, but cannot be determined for all fibers in the motor unit because most are beyond the recording radius of the triggering electrode. Transmission blocking is likely to involve only a small percentage of muscle fibers unless the rare form of neurogenic blocking occurs, which could cause a loss of a greater number of fibers. ${ }^{18}$ Blocking will result in lower amplitude motor unit potentials being included in the S-MUP average, which has been shown to have little effect on the average (Table 1).

Temporal Shifting of Trigger Potentials. When the trigger potential is complex and includes many spike components, variations in the relative amplitudes of the spike components can result in a shift so that different spikes in the potential cross the trigger voltage threshold first (Fig. 4). This results in a temporal shift of the trigger point from one spike to another within the trigger potential. The effect of temporal shifting on the averaged amplitude was investigated in a simulation model (Fig. 6 ). The resultant change in averaged amplitude (Table 2) indicates that a shift of $2 \mathrm{~ms}$ has little effect on the averaged amplitude even when up to $20 \%$ of the averaged potentials are shifted. A shift of $4 \mathrm{~ms}$, however, is unacceptable when more than $5 \%$ of the potentials are so shifted.

Effect of Motor Unit Depth on S-MUP Amplitude. Muscle fibers of a motor unit occupy limited crosssectional areas in a muscle and the area of the motor unit does not expand appreciably with collateral reinnervation in ALS. ${ }^{18}$ Attenuation of the S-MUP signal should be proportional to the distance between the muscle fibers of the motor unit and the surface electrode. The thickness of subcutaneous tissue is an additional attenuating factor. However, no significant correlations were found between S-MUP amplitude and electrode depth (ALS subjects, $P=0.30$; normal subjects, $P=$ 0.26). In the ALS subjects, a macro-EMG potential amplitude was recorded simultaneously from the same motor unit, and a significant correlation between the S-MUP and macro-EMG amplitudes was found $(P=0.0001)$, supporting an accurate representation of motor unit amplitude from the surface recording in ALS subjects.

Trial-to-Trial Variability of S-MUP Amplitude. In a small number of ALS subjects, the trigger potential remained sufficiently stable over several minutes so that the same S-MUP could go through the same spike-triggered averaging process multiple times. The coefficient of variation was less than $10 \%$, supporting an acceptable level of trial-to-trial variability for this MUNE method.

\section{DIscussion}

Recording a maximal CMAP is required for all MUNE methods because it represents the numerator in the MUNE ratio. An erroneous CMAP amplitude can be a major source of error because the CMAP is a single estimate in contrast to the S-MUP value which is an estimate of a mean value. The contribution of volume conduction from neighboring muscles innervated by the same nerve is difficult to estimate. ${ }^{17}$ Examples include the thenar and hypothenar muscle groups with volume conduction from median and ulnar innervated lumbrical muscles, respectively, the anterior tibialis muscle with volume conduction from the peroneal muscle group, and the biceps brachii muscle with volume conduction from the brachialis muscle. ${ }^{9,16}$ The extensor digitorum brevis muscle is remote from other peroneal nerve innervated muscles, and is least affected by volume conduction. ${ }^{13}$ An erroneous CMAP is usually inflated by phase addition of the distant signals, but may also be reduced by phase cancellation. Erroneously large CMAPs will give inflated MUNE values and smaller CMAPs will give reduced values. 
Achieving an accurate average S-MUP response with the spike-triggered averaging method is dependent upon maintaining the same trigger potential. Calculations demonstrate that spurious potentials of high amplitude, more than those of low amplitude, can affect the amplitude of the averaged potential (Table 1). These calculations are based on a formula for a simple running average, the method of averaging used in many EMG machines. EMG machines using methods based on the principle of averaging median values will be less affected by high amplitude spurious potentials. There are other methods to handle spurious triggers, such as recording the entire signal ensemble, identifying the spurious triggers, and editing out the associated S-MUPs from the average either manually or by statistical algorithm. At the present time, these techniques require special computer equipment and software which is not available on EMG machines used in clinical settings.

The spike-triggered averaging MUNE method is based on averaging S-MUPs from voluntary motor units with low recruitment thresholds. In normal subjects, the range of motor unit amplitudes within a muscle is limited, and spurious voluntary potentials will fall within the same range. In ALS subjects, spurious triggers may include high threshold motor units, enlarged motor units, or fasciculation potentials. Alpha motor neuron death in ALS appears to be indiscriminate and is not based on the size of the cell body or the motor unit. $^{15,21}$ The degree or extent of reinnervation is limited in ALS to perhaps a twofold increase in the number of muscle fibers in a motor unit. ${ }^{18} \mathrm{Al}-$ though fasciculation potentials may appear to be large on the oscilloscope screen, the size of the fasciculation potential recorded by the surface electrode is unknown because the potential is within the averaged signal. It is unlikely that the amplitude of the fasciculation potential lies beyond the range of voluntarily recruited units. In support of a size limitation of fasciculation potentials is the finding that the amplitude of the macro-EMG of a fasciculation potential is not different from that recorded from voluntary motor units in ALS subjects, and many fasciculation potentials include only a portion of a motor unit. ${ }^{11}$ Accordingly, the outlying S-MUP amplitude values in the distribution recorded from the biceps-brachialis muscle group in ALS subjects (Fig. 4) likely reflect maximal motor unit amplitudes.

Differences in amplitude between triggered and spurious potentials can be viewed in the following manner. The distributions of S-MUP am- plitudes are skewed in normal and ALS subjects. For both groups, the maximal S-MUP amplitude values are not greater than ten times the mean or median values. Thus, a spurious potential with an outlying amplitude value will be, on average, within one order of magnitude of the amplitude of an average triggered potential.

S-MUPs triggered from deep-lying motor units have been found to be lower in amplitude than superficial units in normal subjects, but the differences have not always been statistically significant. ${ }^{3,8}$ Signal attenuation through muscle and subcutaneous tissue is felt to account for lower amplitudes. ${ }^{3}$ No significant differences or trends were observed in either normal or ALS subjects in this study. The larger size of motor units in ALS subjects likely makes any attenuation a less influential factor especially in this subject population. Additionally, although we did not measure the amount of subcutaneous tissue, it is likely to be less in ALS subjects. It remains important, however, to attempt to trigger on motor units at all depths within a muscle.

Neuromuscular junction transmission, while secure in normal subjects, may fail at higher discharge rates in muscles that have recently undergone reinnervation. ${ }^{18}$ The discharge rate of motor units sampled in the spike-triggered averaging method is high $(10-20 \mathrm{~Hz})$ when compared to the rates in the other MUNE methods which use electrical stimulation of motor nerves $(1-3 \mathrm{~Hz})$. Abnormal neuromuscular junction jitter is frequently observed in the trigger potentials from ALS subjects, and blocking of transmission will reduce the amplitude of the S-MUP. This has been demonstrated to produce a $30 \%$ or greater S-MUP amplitude reduction with short discharge trains. ${ }^{7}$ However, two factors will make it unlikely that transmission failure and fiber dropout will have an appreciable effect on the spike-average method when the trains are longer than 200 discharges. With steady discharge rates, posttetanic potentiation will reduce the magnitude of the decrement, ${ }^{22}$ and the inclusion of lower amplitude responses has been shown to have a minimal effect on the average S-MUP amplitude (Table 1).

Other factors which may induce errors in the spike-triggered averaging method include synchronization of the discharge of several motor units with the trigger potential. Synchronization rarely occurs in normal subjects, ${ }^{14}$ but could be a consideration when there are relatively few motor units remaining, such as in ALS, when a tremorlike pattern of recruitment can be observed. This usu- 
ally occurs with near maximal levels of contraction in severely affected subjects, and grouped discharges can be detected over the audio monitor. In this study, performed at relatively low levels of contraction, synchronized patterns were not observed. The surface signals from entrained motor units could add and inflate the average S-MUP to give an underestimation of the MUNE. However, there is increased variability in the discharge rates of motor units in ALS subjects. ${ }^{15}$ Thus, potential increases the S-MUP amplitude by grouped discharges would be lessened by the temporal shifting back and forth of the spurious potentials, much like the effect of shifting trigger potential presented in Table 2 and Figure 5.

In summary, the spike-triggered averaging method has advantages of availability on many EMG machines and applicability to MUNE in proximal muscles. The sources of error unique to this method relate primarily to spurious and erroneous trigger potentials. With care to monitoring the trigger potentials, these sources can be controlled.

\section{REFERENCES}

1. Andres PL, Hedlund W, Finison L, Conlon T, Felmus M, Munsat TL: Quantitative motor assessment in amyotrophic lateral sclerosis. Neurology 1986;36:937-941.

2. Andres PL, Finison LJ, Conlon T, Thibodeau LM, Munsat TL: Use of composite scores (megascores) to measure deficit in amyotrophic lateral sclerosis. Neurology 1988;38: 405-408.

3. Barkhaus PE, Nandedkar SD: Recording characteristics of the surface EMG electrodes. Muscle Nerve 1994;17:13171323.

4. Bromberg MB: Motor unit estimation: reproducibility of the spike-triggered averaging technique in normal and ALS subjects. Muscle Nerve 1993;16:466-471.

5. Bromberg MB, Forshew DA, Nau KL, Bromberg J, Simmons Z, Fries TJ: Motor unit number estimation, isometric strength, and electromyographic measures in amyotrophic lateral sclerosis. Muscle Nerve 1993;16:1213-1219.

6. Bromberg MB, Fries T, Forshew D, Nau K, Fillya M, Badger G, Carpenter J, Tandan R: Motor unit number estima- tion as an outcome measure in a two-center controlled trial of amion acids in amyotrophic lateral sclerosis [abstract]. Muscle Nerve 1994;17:1100-1101.

7. Brown WF, Jaatoul N: Amyotrophic lateral sclerosis: electrophysiologic study (number of motor units and rate of decay of motor units). Arch Neurol 1974;30:242-248.

8. Brown WF, Milner-Brown HS: Some electrical properties of motor units and their effects on the methods of estimating motor unit numbers. J Neurol Neurosurg Psychiatry 1976; 39:249-257.

9. Brown WF, Strong MJ, Snow R: Methods for estimating numbers of motor units in biceps-brachialis muscles and losses of motor units with aging. Muscle Nerve 1988;11: $423-432$.

10. Doherty TJ, Brown WF: The estimated numbers and relative sizes of thenar motor units as selected by multiple point stimulation in young and older adults. Muscle Nerve 1993;16:355-366.

11. Guiloff RJ, Modarres-Sadeghi H: Voluntary activation and fiber density of fasciculations in motor neuron disease. Ann Neurol 1992;31:416-424.

12. McComas AJ: Invited review: motor unit estimation: methods, results, and present status. Muscle Nerve 1991;14: 585-597.

13. McComas AJ, Fawcett PRW, Campbell MJ, Sica REP: Electrophysiological estimation of the number of motor units within a human muscle. J Neurol Neurosurg Psychiatry 1971; 34:121-131.

14. Milner-Brown HS, Stein RB, Yemm R: The contractile properties of human motor units during voluntary isometric contractions. I Physiol 1973;228:285-306.

15. Petajan JH: Clinical electromyographic studies of diseases of the motor unit. Electroencephalogr Clin Neurophysiol 1974; 36:395-401.

16. Sica REP, McComas AJ, Upton ARM, Longmire D: Motor unit estimations in small muscles of the hand. J Neurol Neurosurg Psychiatry 1974;37:55-67.

17. Slawnych MP, Laszlo CA, Hershler C: A review of techniques employed to estimate the number of motor units in a muscle. Muscle Nerve 1990;13:1050-1064.

18. Stålberg E: Electrophysiological studies of reinnervation in ALS, in Rowland LP (ed): Human Motor Neuron Disorders. New York, Raven Press, 1982, pp 47-59.

19. Stashuk DW, Doherty TJ, Kassam A, Brown WF: Motor unit number estimates based on the automated analysis of F-responses. Muscle Nerve 1994;17:881-890.

20. Stein RB, Yang JF: Methods for estimating the number of motor units in human muscles. Ann Neurol 1990;28: $487-495$

21. Strong MJ, Brown WF, Hudson AJ, Snow R: Motor unit estimates in the biceps-brachialis in amyotrophic lateral sclerosis. Muscle Nerie 1988;11:415-422.

22. Trontelj JV, Stålberg E: Single motor end-plates in myasthenia gravis and LEMS at different firing rates. Mzsscle Nerve 1991;14:226-232. 